\title{
Composition, Chemical Fingerprinting and Antimicrobial Assessment of Costa Rican Cultivated Guavas (Psidium friedrichsthalianum (O. Berg) Nied. and Psidium guajava L.) Essential Oils from Leaves and Fruits
}

\section{Fabio Granados-Chinchilla ${ }^{1 *}$, Erick Villegas ${ }^{1}$, Andrea Molina $^{1,2}$ and Carlos Arias ${ }^{3}$}

${ }^{1}$ Centro de Investigación en Nutrición Animal (CINA), Universidad de Costa Rica, 11501-2060 Ciudad Universitaria Rodrigo Facio San Jose, Costa Rica ${ }^{2}$ Escuela de Zootecnia, Universidad de Costa Rica, 11501-2060 Ciudad Universitaria Rodrigo Facio San José, Costa Rica

${ }^{3}$ Escuela de Química and Centro de Investigación en Productos Naturales (CIPRONA), Universidad de Costa Rica, 11501-2060 Ciudad Universitaria Rodrigo Facio, San José, Costa Rica

\begin{abstract}
The essential oil of two related tree species, $P$. friedrichsthalianum and $P$. guajava, where obtained. A total of six different oil samples were recovered including leaves in dry/rainy season and fruits of both plant species. Oil yields ranged between $0.128 \%$ ( $P$. friedrichsthalianum leaves during dry season)- $0.743 \%$ ( $P$. guajava leaves during rainy season). All extracts were subjected to a GC/MS analysis using, during the chromatographic separation, a polyethylene glycol column. In general terms, we recognized three independent biosynthetic routes i. aromatic compounds ii. Terpenes and iii. Fatty acids derivatives. Several compounds were found to be preserved in the oils, such as 2,4-ditert-butylphenol, $\alpha$-terpineol and neointermedeol whereas Costa Rican guava fruit exhibit unique compounds such as $2 \mathrm{H}$-pyran-2,6-(3H)-dione. Terpenes and fatty acids are among the most variable $(p<0.005)$ in content when comparing dry season with rainy season leaves. Finally, based on profiling, a descriptive PCA analysis showed three related groups and that Costa Rican guava fruit oil as the most different in terms of composition. Herein we report more than 50 compounds for each species and relative percentages of major components $(>0.1 \%)$ and trace compounds. In addition, we evaluated the antimicrobial activity of these essential oils against common foodborne and food-spoilage related bacteria. The rainy season $P$. guajava leafs' presented the highest antimicrobial activity against all the bacteria strains tested, with inhibition zones ranging from 31 to $52 \mathrm{~mm}$. This study will help understand volatile composition of a fruit producing plant native from this geographic area and hints toward possible applications.
\end{abstract}
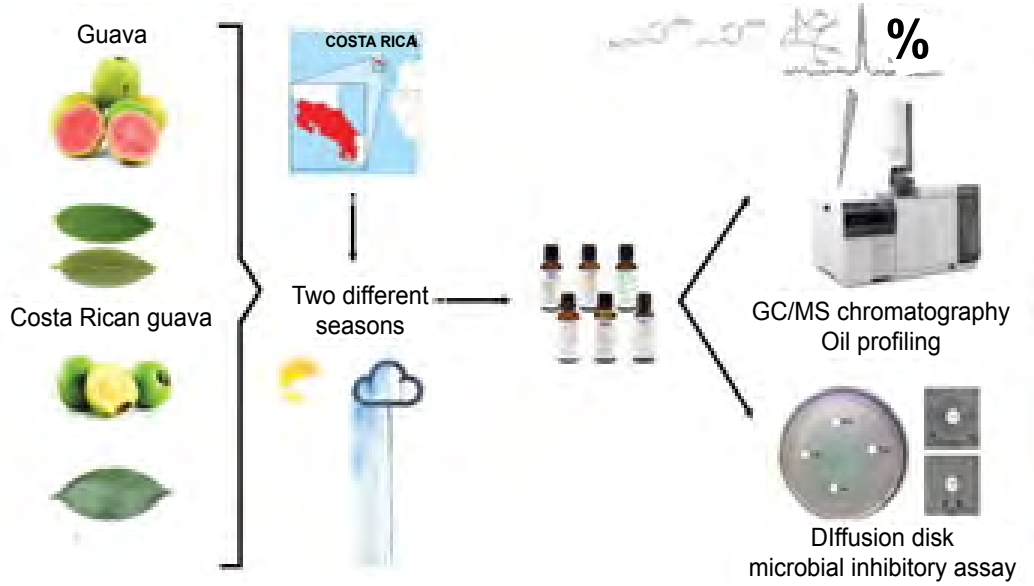

Keywords: Psidium friedrichsthalianum; Psidium guajava; Essential oil; Volatile compounds; GC/MS

\section{Introduction}

The Myrtaceae is a family of dicotyledonous plants which is comprised of at least 5650 species (ca. 130-150 genera) [1,2]. One group of trees and shrubs contained in this family, Psidium, are native to warmer parts of the Western Hemisphere [3]. Specifically, two economically and nutritionally relevant species in Costa Rica are P. friedrichsthalianum (found in Southern Mexico and Central America) commonly known as Costa Rican guava [4] and P. guajava (found in Central and South America, West Indies, Mexico, Florida, Louisiana, Arizona) [5].

Essential oils are usually by-products of fruits or fruit tree processing [6]; their importance reflects their industrial or bioactive properties [7]. Furthermore, as Costa Rica's tropical fruit production and exportation (estimated at 1600 million USD in 2014) has increased in the last several years [8], so has fruit processing to juice and pulp.
Extraction of essential oils from such species is not only feasible, but also represents a viable alternative to increase value from the fruit production industry [9]. In 2011 alone, the essential oil global industry was estimated to be ca. 24 billion USD [9].

*Corresponding author: Fabio Granados-Chinchilla, Centro de Investigación en Nutrición Animal, Universidad de Costa Rica, 11501-2060 Ciudad Universitaria Rodrigo Facio San José, Costa Rica, Tel: +50625112028; E-mail: fabio.granados@ucr.ac.c

Received July 02, 2016; Accepted July 22, 2016; Published July 28, 2016

Citation: Granados-Chinchilla F, Villegas E, Molina A, Arias C (2016) Composition, Chemical Fingerprinting and Antimicrobial Assessment of Costa Rican Cultivated Guavas (Psidium friedrichsthalianum (O. Berg) Nied. and Psidium guajava I.) Essential Oils from Leaves and Fruits. Nat Prod Chem Res 4: 236 doi:10.4172/2329-6836.1000236

Copyright: (c) 2016 Granados-Chinchilla F, et al. This is an open-access article distributed under the terms of the Creative Commons Attribution License, which permits unrestricted use, distribution, and reproduction in any medium, provided the original author and source are credited. 
Citation: Granados-Chinchilla F, Villegas E, Molina A, Arias C (2016) Composition, Chemical Fingerprinting and Antimicrobial Assessment of Costa Rican Cultivated Guavas (Psidium friedrichsthalianum (O. Berg) Nied. and Psidium guajava I.) Essential Oils from Leaves and Fruits. Nat Prod Chem Res 4: 236. doi:10.4172/2329-6836.1000236

Page 2 of 10

Because the quality and composition of essential oils depends on different factors such as plant chemo type and biotype as well as the climatic conditions [10,11], a study of the influence of different periods of ripening on the chemical fingerprinting of guava and Costa Rican guava essential oil from leaves is, therefore, considered useful. A similar approach has been used to characterize essential oil of other plants. Despite the relevance of such Psidium species, the volatile compounds of both the leaves and fruits of $P$. friedrichsthalianum have only partially been described [12], whereas $P$. guajava volatiles have been described in regions where the tree is not native [13]. The complete chemical composition of essential oils from other Psidium species has been described elsewhere $[14,15]$.

It is well known that many volatile compounds found in oil bearing plants are implicated in plant defense and cytotoxic activity against pathogens and/or fungi $[16,17]$. The chemical species related to the volatile components that may be responsible for this activity are seldom addressed.

To our knowledge there is no information regarding the composition of these trees grown in their native Central America region and no literature sources have compared both Psidium species leaves and fruits including leaf's oil composition changes with respect to any edaphoclimatic conditions. Herein, we describe the essential oils components of leaf (in dry and rainy seasons) and fruit from $P$. friedrichsthalianum and P. guajava cultivated in Costa Rica and explore their antimicrobial potential against common foodborne pathogens.

\section{Materials and Methods}

\section{Plant material and extraction}

$P$. friedrichsthalianum and P. guajava leaves were collected in two different weather conditions (dry and rainy) during the months of April (average of $11.3 \mathrm{~mm}$ precipitation and 4 days of rain) and July (average of $223.0 \mathrm{~mm}$ precipitation and 23 days of rain) respectively, from local areas of San José, Costa Rica. Only undamaged leaves were collected. Mature fruits were collected directly from the tree when in season. Specimens were identified and selected based on structural characteristics of their leaves and trunks by a biologist with botanical and taxonomical expertise and based on the guidelines previously described by Sharma et al. [18]. All samples were collected from adult trees and randomly from the tops. Each collection was formed by sampling three different specimens. The essential oil was extracted by the process of steam distillation using an all glass still and purified water. Briefly, crushed fruits and aerial parts of plant material (ca. 150 $\mathrm{g}$ in each case) were placed in a Clevenger type apparatus with 1000 $\mathrm{mL}$ flask, oil separator tube and condenser, $250 \mathrm{~mL}$ of purified water was added and the mixture was vapor distilled (at $96^{\circ} \mathrm{C}$ at a rate of $20^{\circ} \mathrm{C} /$ minute and then kept at $96^{\circ} \mathrm{C}$ for 180 minutes) into a $125 \mathrm{~mL}$ Erlenmeyer, which was used to collect the aqueous distillate. The receiving receptacle was kept cold $\left(0^{\circ} \mathrm{C}\right.$, using acetone-ice mixture) during the extent of distillation. Finally, liquid-liquid extraction was performed, with diethyl ether as the organic solvent, in order to recover volatiles. The organic fraction was dried in a rotatory evaporator until an oily substance (invariably, mixtures of organic volatile compounds) was obtained. Only ripened, healthy (without visible scarring) and non-infested (by common pests such as members of the Tephritidae family) [19], fruits were processed. In the samples of fruits, paraffin was added to avoid foaming of the non-volatile material in the flask during processing. Type III water with a final conductivity of $<10 \mu \mathrm{Scm}^{-1}$ was obtained using a RiOS ${ }^{\mathrm{TM}}$ system (EMD Millipore, Billerica, MA, USA). Oil yields ranged between $0.13 \%$ (P. friedrichsthalianum leaves during dry season) $-0.74 \%$ ( $P$. guajava leaves during rainy season).

\section{GC/MS analysis}

Qualitative analyses of the volatile compounds were carried out by means of an Agilent gas chromatography (Agilent Technologies, Santa Clara, CA) equipped with an Agilent Technologies J\&W DBWAX micro bore column of $10 \mathrm{~m}$ length, $0.1 \mathrm{~mm}$ diameter, $0.1 \mu \mathrm{m}$ film thickness and Agilent 5977E mass spectrometer (MSD). The carrier gas was helium at a constant flow of $0.3 \mathrm{~mL} / \mathrm{min}$. The GC oven temperature was kept at $50^{\circ} \mathrm{C}$ for 0.34 minutes and programmed to $200^{\circ} \mathrm{C}$ at a rate of $72.51^{\circ} \mathrm{C} /$ minute, this temperature was kept constant 0.17 minutes and then programmed to $230^{\circ} \mathrm{C}$ at a rate of $8.7^{\circ} \mathrm{C} /$ minute, held for 7.9 minutes for a total run time of $13.93 \mathrm{~min}$. The split ratio was adjusted at 30:1. The injector temperature was set at $250^{\circ} \mathrm{C}$. The mass range was $50-450 \mathrm{~m} / \mathrm{z}$. Electron energy was set at $70 \mathrm{eV}, 150^{\circ} \mathrm{C}$. Constituents were identified by matching their spectra with those in NIST library 14 . Only hits with a match factor above $80 \%$ were considered. In all cases geraniol (98\%, 163333, Sigma-Aldrich, St Louis, Mo) was used as an internal standard. Additionally, transcinnamic $\operatorname{acid}_{(7.28 \mathrm{~min} ; \mathrm{M}+149.0 \mathrm{~m} / \mathrm{z})^{\prime}}$, benzeneacetic $\operatorname{acid}_{(5.64 \mathrm{~min} ; \mathrm{M}+135.2 \mathrm{~m} / \mathrm{z})^{\prime}}$ benzoic $\operatorname{acid}_{(5.07 \mathrm{~min} ; \mathrm{M}+121.3 \mathrm{~m} / \mathrm{z})}, 2$,4-ditertbutylphenol $(4.69 \mathrm{~min} ; \mathrm{M}+207.3 \mathrm{~m} / \mathrm{z})^{\prime}$, globulol $_{(4.07 \mathrm{~min} ; \mathrm{M}+223.4 \mathrm{~m} / \mathrm{z})}$, caryophyllene oxide ${ }_{(3.81 \mathrm{~min} ; \mathrm{M}+220.1 \mathrm{~m} / \mathrm{z})}$, benzyl alcohol $_{(3.46 \mathrm{~min} ; \mathrm{M}+109.1 \mathrm{~m} / \mathrm{z})}, \boldsymbol{a}$-terpineol ${ }_{(2.92 \mathrm{~min} ; \mathrm{M}+155.3 \mathrm{~m} / \mathrm{z})}$, caryophyllene $_{(2.65}$ $\left.\min ; M_{+} 205.4 \mathrm{~m} / \mathrm{z}\right)$, linalool $_{(2.46 \mathrm{~min} ; \mathrm{M}+136.3 \mathrm{~m} / \mathrm{z})}$, benzylaldehyde $_{(2.37 \mathrm{~min} ; \mathrm{M}+107.1 \mathrm{~m} / \mathrm{z})^{\prime}}$,

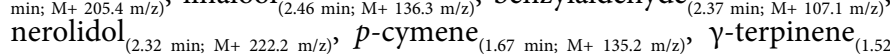

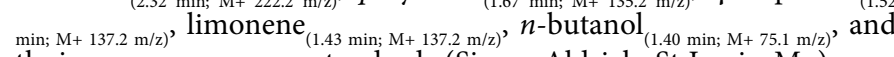
thujone ${ }_{(1.05 \mathrm{~min} ; \mathrm{M}+153.1 \mathrm{~m} / \mathrm{z})}$ standards (Sigma-Aldrich, St Louis, Mo) were injected separately for confirmation purposes. Tetradecanoic ${ }_{(6.16 \mathrm{~min} ; \mathrm{M}+}$ $227.6 \mathrm{~m} / \mathrm{z})$, pentadecanoic ${ }_{(6.72 \mathrm{~min} ; \mathrm{M}+243.4 \mathrm{~m} / \mathrm{z})}$, hexadecanoic $(7.58 \mathrm{~min} ; \mathrm{M}+256.3 \mathrm{~m} / \mathrm{z})^{\prime}$ octadecanoic $_{(9.70 \mathrm{~min} ; \mathrm{M}+285.5 \mathrm{~m} / \mathrm{z})}$, cis-13-octadecanoic ${ }_{(10.21 \mathrm{~min} ; \mathrm{M}+285.7 \mathrm{~m} / \mathrm{z})}$ and 9Z-octadecenoic ${ }_{(7.78 \mathrm{~min} ; \mathrm{M}+284.1 \mathrm{~m} / \mathrm{z})},(Z, Z)-9,12$-octadecadienoic $(10.86 \mathrm{~min} ; \mathrm{M}+$ acids were obtained from (Nu-Chek Prep, Inc., Elysian, MN, USA). Analytes with $\geq 5 \%$ relative composition, and when available commercially, were simultaneously monitored by SIM mode (total dwell time $100 \mathrm{~ms}$ and cycles $8.3 \mathrm{~Hz}$ ) using the ions and retention times specified above. For compounds with no analytical standard injection, identification should be considered as tentative.

\section{In vitro antimicrobial activity}

Each of the six essential oils were mixed 80:20 with dimethyl sulfoxide (DMSO, Sigma-Aldrich, St Louis, Mo) and homogenized. Aliquots of $10 \mu \mathrm{L}$ of these extracts were pipetted onto sterile discs of $7 \mathrm{~mm}$ of diameter prepared with 934-AH Whatman glass micro fiber filters (Whatman International Ltd, Maidstone, UK), the discs were placed onto $4 \mathrm{~mm}$ height Mueller-Hinton agar plates containing $10^{4}$ $10^{6} \mathrm{CFU} \mathrm{mL} \mathrm{mL}^{-1}$ of the following strains: S. Choleraesuis ATCC 10708, S. typhimurium ATCC 14028, S. enteritidis ATCC 13076, E. coli O157:H7 ATCC 43888, S. aureus subsp. Aureus ATCC 25923, B. cereus ATCC $13061, B$. subtillis ATCC 11774, P. aeruginosa ATCC 27853, $P$. mirabilis ATCC 25933 and E. coli ATCC 25922. These experiments were performed by triplicate. In addition, discs impregnated with water were tested in parallel to confirm that the filter paper used in their manufacturing was not toxic to E. coli ATCC 25922. An aqueous 10 $\mu \mathrm{g} \mathrm{mL}{ }^{-1}$ solutions of oxytetracycline and DMSO were used as positive and negative controls, respectively. Additionally, some essential oil standards were tested in parallel for comparison (i.e., limonene, myrcene, terpinene, eucalyptol, linalool, thujone, caryophylene and cymene;purchased from Sigma-Aldrich, St Louis, Mo).

\section{Statistical analysis}

ANOVA analysis with post-hoc Tukey test was used to explore statistical differences in relative concentrations among major components in the six different essential oils. Likewise, a categorization 
of components based on their structural similarities was as follows: aromatic compounds, terpenes, fatty acids (and derivatives) and linear aliphatic hydrocarbons. The same test was used to analyze differences between relative concentrations obtained for the aforementioned categories. Principal component analysis was performed to the chemical fingerprint of the six oils in order to assess correlation, if any, among compositions. Components considered relevant if values were above $|0.4|$ in the rotated matrix. All assays carried out using IBM SPSS Statistics version 22 (SPSS, Inc., Armonk, NY, USA).

\section{Results and Discussion}

The chemical profile obtained for the essential oils resulted to be a complex mixture as evidenced by heavily signal-charged total ion chromatograms (TIC). The chromatogram of P. guajava fruit essential oil serves as an example and is presented in Table 1 and Figures 1 and 2.

In the case of $P$. friedrichsthalianum leaf oil, caryophyllene (Table 1) was found to be only a minor component of the mixture $(1.87 \%)$; this result may be a biological response to direct sunlight perceived by the plant as it has been demonstrated in other oils in a tropical country [20-22] as well as other geochemical factors (e.g., soil type and precipitation) [23].

Based in the data gathered here, we recognized three independent biosynthetic routes i) aromatic compounds ii) Terpenes and iii) Fatty acids derivatives. Aromatic compounds (i.e., phenols, benzenoids and phenylpropenes) may be present to preserve antioxidant capacity in green leaves in case of mechanical shear, stress or injury [24]. The presence of these antioxidants in larger quantities, in Costa Rican guava leaves, may also be due to the fact that Psidium species are deciduous trees, hence the moment of the sampling may correlate with a mayor leaf shedding process which may be assisted by such a compound. Interestingly, these compounds are also present in guava fruit.

In the case of leaf oil, compounds containing a tert-butyl moiety (i.e., 2,4,6-Tris(1,1-dimethylethyl)-4-methylcyclohexa-2,5-dien-1one; 2,3-dimethyl-5-oxohexanethioic acid S-t-butyl ester; 2,4-ditert-butylphenol)occur repeatedly especially in phenol and quinone based structures. This may very well be part of a reaction blockage or protection (avoid premature reactivity) mechanism [25] to preserve synthesized compounds needed downstream from the biogenic pathway.

Among the sulfur containing compounds found, of special interest is 2-hydroxy-3-(thiophene-2-yl)methyl-5-methoxy-1,4-benzoquinone. In general, functionalized quinones have been already described as compounds of interest due to their potential as antimalarial drugs [26]. Synthesis should be pursued.

The major components ( $>3 \%$ ) of dry season Costa Rican guava leaves essential oil were: 2,4-di-tert-butylphenol [1] (27.62\%), $a$-terpineol [2] (10.53\%), neointermedeol (9.96\%), 2-Hydroxy-3-(thiophen-2-yl) methyl-5-methoxy-1,4-benzoquinone (6.80\%) caryophyllene oxide (3.43\%) and globulol (3.33\%). 2,4-di-tert-butylphenol [1] isolated from other natural sources (for example sweet potato [27]) is known as a compound with oxidative stress protection capabilities. The presence of this substance, in considerable percentages in other Myrtacea essential oils, [28] has been described previously. The antioxidative efficiency of phenolic compounds is increased when t-butyl groups are located in positions 2,4 and 6 of the aromatic ring [29]. In an analogous manner, 2H-pyran-2,6-(3H)-dione [6] has been also extracted from other oil bearing fruits such as Triphala in considerable quantities [30].

Furthermore, $\alpha$-terpineol already has been described as an NF$\mathrm{\kappa B}$ signaling suppressor [31] and gastroprotective activity in animal models [32]. This should be noted that $a$-terpineol has been found in all $P$. friedrichstalianum oils with invariably relative concentrations of $\geq 10 \%$ (Figure $3 \mathrm{~A}$ ).

On the other hand, the major components of dry season guava leaves essential oil were: neointermedeol [3] (19.5\%), 7-epi- $\alpha$-selinene [9] (17.0\%), nerolidol [7] (9.5\%); caryophyllene (9.3\%), 10,10-dimethyl2,6-dimethylenebicyclo[7.2.0] undecan-5 $\beta$-ol (8.4\%), benzaldehyde (5.5\%), caryophyllene oxide (8.1\%), benzyl alcohol (4.5\%).

Benzoic acid [5] a relatively common compound in oils [29] and a product of shikimate aromatization pathway, was found to be a major component in $P$. fridrichstalianum dry season leaf essential oil, and may serve as a building block (through amination or hydroxylation) for biosynthesis of more significant compounds [33]. This is reinforced by the capability of benzoic acid to eventually form derivatives (e.g., esters, aldehydes, phenols). In fact, some of these derivatives may already be found (e.g., 4-benzyloxybenzoic acid, 2-hydroxyethyl benzoate, benzaldehyde, benzyl alcohol). In fact, these derivatives have been found to be even more common in essential oils that their parent compound [33]. This may hint towards a more biosynthetically active plant when in rainy season.

As expected, some similarities do arise between both species when grown in the same region, under similar conditions and when leaves are harvested in the same season. Though in different compositions, several compounds are found in both tree leaves such as linalool, caryophyllene [8] and its oxide, neointermedeol [3] and a-terpineol [2] to name just a few (Table 1). It would appear these biosynthetic compounds are conserved and hence their synthetic routes [34]. For example, invariably, a-terpineol [2] was a constant compound recovered from Psidium essential oils. Interestingly, 2,4-di-tertbutylphenol [1] was also found in guava leaves collected in dry season, however its amount was negligible. Costa Rican Guava leaves extract exhibited, under the same extraction conditions, a mixture of fewer compounds (Table 1).

Further research may be focused on the characterization of these essential oils in order to determine biological activity such as antifungal, antimicrobial or antioxidant capability and exploit these characteristics, if any, by means of an application for animal or human nutrition, especially since this oils are generally regarded as safe (GRAS) [35]. For example, other researchers have evaluated Psidium leaves potential as forage [36], tried to incorporate the leaf meal or crude extract of $P$. guajava into broiler chicken diets [37] and even have introduced them into rat diets [38].

In all cases, the four primary compounds found in the extract described invariably $>40 \%$ of the composition (Figures $3 \mathrm{~A}$ and $3 \mathrm{~B}$ ). The repeated major components found among the six oils showed significantly different percentages (Figures $3 \mathrm{~A}$ and $3 \mathrm{~B}$ ). Furthermore, when the chemical profiles are analyzed as four different groups of compounds, fruits of both species show rather similar compositions (Figures 4A and 4B). Marked differences are however evident when comparing chemical families among leaf essential oil from both species. The more interesting characteristics found were a dramatic drop $(p<0.05)$ in terpenes in contrast to an increase in fatty acids when comparing $P$. guajava leaves in dry and rainy season (Figure $4 \mathrm{~B})$. Evidence of higher amount of terpenes in essential oil from leaves collected in dry season for guava is reinforced by the presence of the tertiary sesquiterpene alcohol nerolidol [7] in important concentrations (9.5\%). Interestingly, this compound has been associated with ripening in other fruits [39]. The compounds 7-epia-selinene and caryophyllene and its oxide suffer from a decrease in 
Citation: Granados-Chinchilla F, Villegas E, Molina A, Arias C (2016) Composition, Chemical Fingerprinting and Antimicrobial Assessment of Costa Rican Cultivated Guavas (Psidium friedrichsthalianum (O. Berg) Nied. and Psidium guajava I.) Essential Oils from Leaves and Fruits. Nat Prod Chem Res 4: 236. doi:10.4172/2329-6836.1000236

Page 4 of 10

\begin{tabular}{|c|c|c|}
\hline \multicolumn{3}{|c|}{ P. friedrichsthalianum } \\
\hline Leaf (dry season) & Leaves (rainy season) & Fruit \\
\hline \multicolumn{3}{|c|}{ Major components } \\
\hline 2,4-di-tert-butylphenol (4.69) [27.6\%] & 2,4-di-tert-butylphenol (4.62) [23.2\%] & 2H-pyran-2,6-(3H)-dione (3.86) [26.4\%] \\
\hline a-terpineol (2.92) [10.5\%] & a-terpineol (2.83) [18.4\%] & cis-13-octadecenoic acid (10.21) [14.0\%] \\
\hline neointermedeol (4.59) [10.0\%] & Tetra decanoic acid (6.16) [9.5\%] & a-terpineol (2.86) [11.7\%] \\
\hline $\begin{array}{c}\text { 2-hydroxy-3-(thiophen-2-yl)methyl-5-methoxy-1,4- } \\
\text { benzoquinone (4.63) [6.8\%] }\end{array}$ & benzoic acid (5.07) [9.2\%] & n-hexadecanoic acid (7.58) [9.6\%] \\
\hline globulol (4.07) [3.3\%] & methyl formate (2.41) [4.7\%] & ammonium acetate (2.50) [5.64\%] \\
\hline caryophyllene oxide (3.81) [3.4\%] & oleic acid $(7.78)$ [4.4\%] & octadecanoic acid (9.79) [5.3\%] \\
\hline spathulenol (4.19) [2.5\%] & 1-nonadecene (3.89) [3.4\%] & benzeneacetic acid (5.64) [5.2\%] \\
\hline elemicin $(5.03)$ [2.2\%] & 1-docosene (3.30) [2.9\%] & N-phenylacetamide (5.64) [4.8\%] \\
\hline methyl octadecyl ether (5.68) [2.2\%] & cetene $(2.45)[2.4 \%]$ & 1-butanol (1.40) [4.5\%] \\
\hline Y-cadinene (4.41) [2.1\%] & nerolidol (2.32) [2.3\%] & trans-cinnamic acid (7.22) [3.8\%] \\
\hline ledene oxide-(II) (4.97) [1.5\%] & 2-nonadecene (4.48) [2.1\%] & 2-furancarboxylic acid (5.12) [2.6\%] \\
\hline isoaromadendrene epoxide (5.16) [1.4\%] & oleic acid 3-hydroxypropyl ester (9.16) [2.0\%] & hexanoic acid (3.31) [1.5\%] \\
\hline nerolidol (3.89) [1.3\%] & ledene $(4.20)$ [1.9\%] & octanoic acid (3.96) [1.2\%] \\
\hline $\begin{array}{l}\text { 2-((2R,4aR,8aS)-4a-methyl-8- } \\
\text { methylenedecahydronaphthalen-2- } \\
\text { yl) prop-2-en-1-ol }(5.21)[1.0 \%]\end{array}$ & 1-octadecanol (5.67) [1.8\%] & palmitoleic acid $(7.80)[0.9 \%]$ \\
\hline 2,6-dimethylnaphthalene (5.20) [1.0\%] & $\beta$-selinene (2.75) [1.8\%] & Z-3-hexen-1-ol (1.99) [0.8\%] \\
\hline (E)-hexen-3-ol (2.04) [0.9\%] & terpinen-4-ol (2.52) [1.6\%] & 3-metil-1-butanol (1.55) [0.7\%] \\
\hline $\begin{array}{l}(1 \mathrm{R}, 7 \mathrm{~S}, E)-7 \text {-isopropyl-4,10- } \\
\text { dimethylenecyclodec-5-enol }(4.26)[0.9 \%]\end{array}$ & 2,6-di-tert-butylbenzoquinone (3.16) [1.3\%] & $\begin{array}{l}\text { 4-hidroxi- } \alpha, \alpha, 4-\text { trimetylcyclohexane methanol (4.11) } \\
{[0.5 \%]}\end{array}$ \\
\hline $\begin{array}{l}\text { 7,9-di-tert-butyl-1-oxaspiro (4,5)-deca- } \\
\text { 6,9-diene-2,8-dione (6.49) [0.8\%] }\end{array}$ & & vanillin (5.72) [0.3\%] \\
\hline cembrene (4.84) [0.8\%] & (Z)-3-hexen-1-ol (1.97) [1.3\%] & 1,1-dimethoxy-2-propanone (3.43) [0.3\%] \\
\hline 1-octadecanol (3.93) [0.8\%] & $\begin{array}{c}\text { (1S,4S,4aS)-1-isopropyl-4,7-dimethyl-1,2,3,4,4a,5- } \\
\text { hexahydronaphthalene (4.34) [1.1\%] }\end{array}$ & tetradecanoic acid (6.20) [0.3\%] \\
\hline$n$-hexadecanoic acid (7.46) [0.6\%] & benzoyl benzyl disulfide (5.85) [1.0\%] & hydroquinone $(8.78)$ [0.3\%] \\
\hline phytol (5.82) [0.5\%] & $\begin{array}{l}\text { 2-(4a,8-dimethyl-2,3,4,5,6,7-hexahydro-1H-naphthalen-2- } \\
\text { yl) propan-2-ol (4.26) [0.9\%] }\end{array}$ & \\
\hline \multicolumn{3}{|l|}{ 1-tridecene (2.66) [0.4\%] } \\
\hline \multicolumn{3}{|l|}{ (E)-4-oxohex-2-enal (3.17) [0.4\%] } \\
\hline \multicolumn{3}{|l|}{ 1-nonadecene (3.30) [0.4\%] } \\
\hline \multicolumn{3}{|l|}{ myrtenol (3.21) [0.3\%] } \\
\hline \multicolumn{3}{|l|}{ octadecanoic acid (9.56) [0.3\%] } \\
\hline \multicolumn{3}{|l|}{ 7-methoxycoumarin (7.88) [0.3\%] } \\
\hline \multicolumn{3}{|c|}{ Trace compounds (i.e., $<0.1 \%$ ) } \\
\hline isobutyl ether (2.13) & thujene $(1.08)$ & 4-methyl-2-oxetanone (1.12) \\
\hline di-tert-butyldicarbonate (2.57) & methylyclooctane (1.47) & 3-penten-2-one (1.33) \\
\hline trans-linalool oxide (6) acetate $(2.71)$ & (Z)-7-tetradecene (1.86) & Y-terpinene (1.45) \\
\hline $\begin{array}{l}\text { 2,3-dimethyl-5-oxohexanethioic } \\
\text { acid, S-t-butyl ester (2.79) }\end{array}$ & 1,2-ethanediol, monoformate (2.51) & 2-methyl-aziridine (1.55) \\
\hline trans-pinocarvyl acetate (2.81) & methoxy-phenyl oxime (3.02) & 4-carene $(1.63)$ \\
\hline $\begin{array}{l}\text { phosphinic acid, diethyl-, methyl } \\
\text { ester (3.47) }\end{array}$ & 1,2,4,5-tetrazin-3-amine (3.17) & 1-methyl-3-(1-methylethyl) benzene (1.64) \\
\hline $\begin{array}{l}\text { 2,4,6-tris(1,1-dimethylethyl)-4- } \\
\text { methylcyclohexa-2,5-dien-1-one (3.50) }\end{array}$ & $\begin{array}{c}\text { 2,4,6-tris(1,1-dimethylethyl)-4-methylcyclohexa-2,5-dien- } \\
\text { 1-one (3.47) }\end{array}$ & Methyl vinyl ketone (1.66) \\
\hline dimethyl sulfone (3.60) & 1H-pyrazolo[3,4-d]pyrimidin-4-amine (3.64) & 1,3-dioxol-2-one (1.73) \\
\hline T-caudinol (4.32) & 3-methylpiridazine (3.76) & propylcyclopropane (1.89) \\
\hline isospathulenol (4.50) & (E)-tetradec-2-enal (3.84) & acetate 4-hexen-1-ol (1.93) \\
\hline phenylethyl alcohol (4.50) & 1,2-dimethyl-azetidine (4.29) & a-methylstyrene (2.35) \\
\hline $\begin{array}{l}\text { 2-hydroxy-3-(thiophen-2-yl) methyl-5- } \\
\text { methoxy-1,4-benzoquinone (4.63) }\end{array}$ & N-tert-butylhydroxylamine (4.36) & 4-methyl-1-(1-methylethyl)-R-3-cyclohexen-1-ol (2.52) \\
\hline $\begin{array}{l}\mathrm{N} \text {-(2,6-dimethylphenyl)-N-[(2E)-3- } \\
\text { methyl-1,3-thiazinan-2-ylidene] amine (5.40) }\end{array}$ & $\mathrm{N}$-methoxymethamine (4.76) & 3-hydroxypropanenitrile (2.52) \\
\hline $\begin{array}{l}\text { bis-benzenamine, } 4,4 \text { '-[(1-methylethylidene) } \\
\text { bis(4,1- phenyleneoxy)] (5.95) }\end{array}$ & 3-(1'-pyrrolidinyl)-2-butanone (4.92) & 1-methyl-4-(1-methylethenyl) cyclo hexanol (2.64) \\
\hline 1-hexadecanol (5.97) & isoelemicin (4.96) & benzeneacetaldehyde (2.69) \\
\hline $\begin{array}{l}\text { 1,7-dimethyl-4-(1- } \\
\text { methylethyl)cyclodecane (7.08) }\end{array}$ & 3,5-di-tert-butyl-4-hydroxybenzaldehyde (5.34) & diphenyl propanetrione (2.73) \\
\hline 45 compounds & N-phenylacetamide (5.70) & $\begin{array}{l}\text { N-propargyloxycarbonyl L-alanine } \\
\text { hexyl ester (2.83) }\end{array}$ \\
\hline
\end{tabular}


Citation: Granados-Chinchilla F, Villegas E, Molina A, Arias C (2016) Composition, Chemical Fingerprinting and Antimicrobial Assessment of Costa Rican Cultivated Guavas (Psidium friedrichsthalianum (O. Berg) Nied. and Psidium guajava I.) Essential Oils from Leaves and Fruits. Nat Prod Chem Res 4: 236. doi:10.4172/2329-6836.1000236

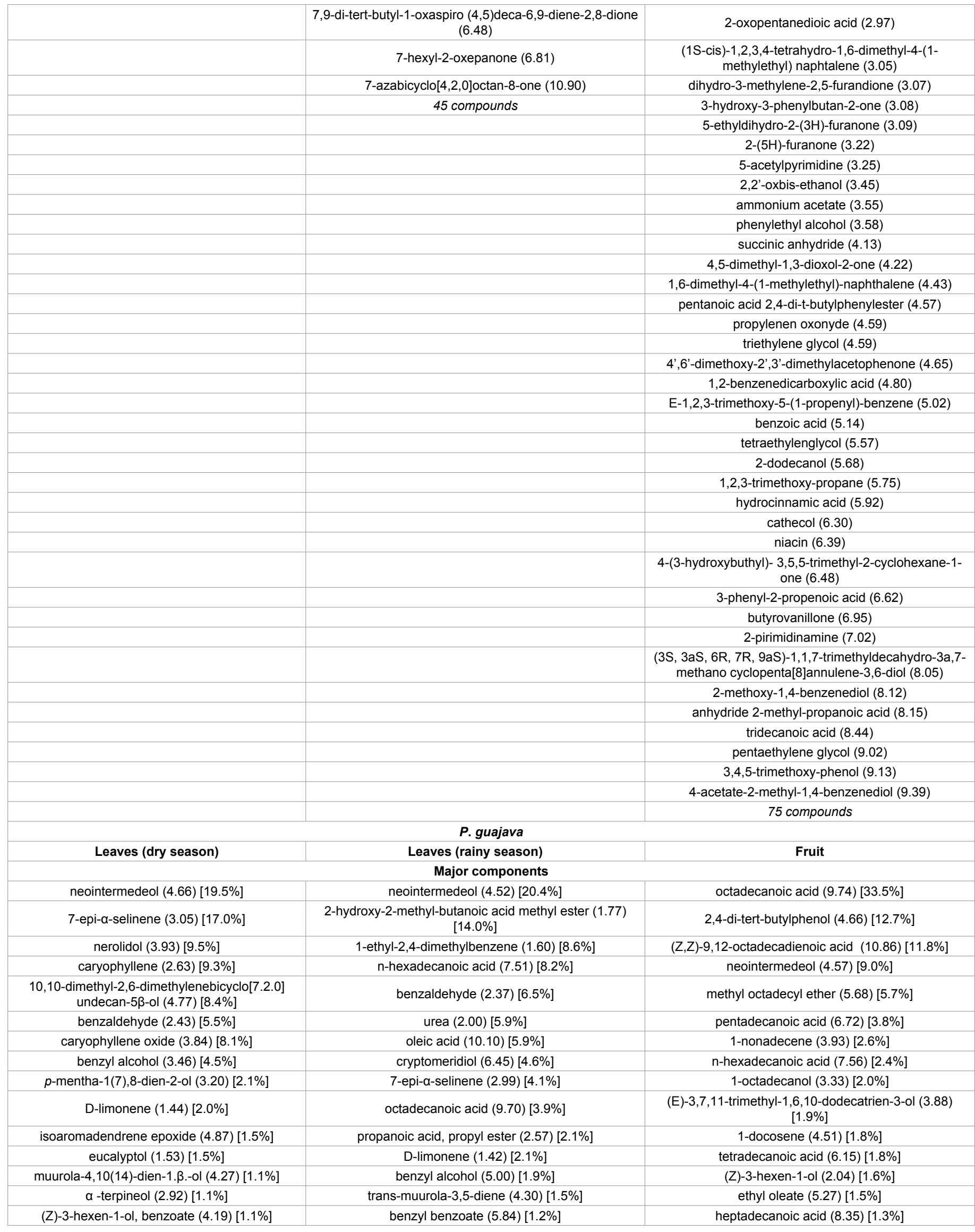


Citation: Granados-Chinchilla F, Villegas E, Molina A, Arias C (2016) Composition, Chemical Fingerprinting and Antimicrobial Assessment of Costa Rican Cultivated Guavas (Psidium friedrichsthalianum (O. Berg) Nied. and Psidium guajava I.) Essential Oils from Leaves and Fruits. Nat Prod Chem Res 4: 236. doi:10.4172/2329-6836.1000236

Page 6 of 10

\begin{tabular}{|c|c|c|}
\hline methyl salicylate $(3.15)[1.0 \%]$ & 9,12-octadecadienoic acid (Z,Z)- (10.80) [1.1\%] & oleic acid $(7.77)[1.3 \%]$ \\
\hline $\begin{array}{c}(1 Z, 4 Z, 7 Z)-1,5,9,9 \text {-tetramethyl-cycloundecatriene } \\
(2.84)[1.0 \%]\end{array}$ & $\begin{array}{l}\text { 11,11-dimethyl-4,8-dimethylenebicyclo [7.2.0]undecan-3- } \\
\text { ol (4.61) [0.9\%] }\end{array}$ & 2,3,3-trimethyl-cyclobutanone (3.16) $[0.8 \%]$ \\
\hline carveol (3.31) [0.9\%] & crotonic anhydride (4.51) [0.7\%] & $\begin{array}{c}\text { [4aR-(4aa, } 7 \alpha, 8 a \beta)]-d e c a h y d r o-4 \alpha-m e t h y l-1-m e t h y l e n e- \\
\text { 7-(1-methylethenyl)- naphthalene }(2.85)[0.8 \%]\end{array}$ \\
\hline cis-Z-a-bisabolene epoxide (6.73) [0.8\%] & 1,3-dioxolane-2-methanol (1.15) [0.7\%] & glycerin $(4.80)[0.7 \%]$ \\
\hline (Z)-3-hexen-1-ol (2.03) [0.7\%] & 1-methyl-4-(1-methylethenyl)-cyclohexanol, $(2.75)[0.7 \%]$ & 2-methyl-1-undecanol (2.62) [0.7\%] \\
\hline I-calamenene (3.33) [0.6\%] & acetophenone (2.82) [0.7\%] & cetene $(2.57)[0.6 \%]$ \\
\hline 11-octadecenoic acid, methyl ester (5.16) [0.4\%] & $\begin{array}{c}\text { 4a,8-dimethyl-2-(prop-1-en-2-yl)-1,2,3,4,4a,5,6,7- } \\
\text { octahydronaphthalene (2.85) [0.6\%] }\end{array}$ & $\begin{array}{l}\text { 2,6-bis(1,1-dimethylethyl)-4-hydroxy-4-methyl- } \\
\text { 2,5-cyclohexadien-1-one (4.06) [0.6\%] }\end{array}$ \\
\hline$\alpha$-limonene diepoxide (6.44) [0.4\%] & $\begin{array}{c}\text { (1S-cis)-1,2,3,4-tetrahydro-1,6-dimethyl-4-(1-methylethyl) } \\
\text { naphthalene (3.27) [0.6\%] }\end{array}$ & $\begin{array}{l}\text { methyl (Z)- } \mathrm{N} \text {-hydroxybenzenecarboximidate (3.05) } \\
{[0.5 \%]}\end{array}$ \\
\hline hexadecanoic acid, methyl ester (4.36) [0.3\%] & terbutol $(3.30)[0.6 \%]$ & octadecanoic acid, ethyl ester (5.19) [0.5\%] \\
\hline phenol, 3,5-bis(1,1-dimethylethyl)- (4.70) [0.3\%] & caryophyllene oxide (4.86) [0.6\%] & \\
\hline n-hexadecanoic acid (7.48) [0.3\%] & tetradecanoic acid $(6.12)[0.5 \%]$ & \\
\hline$\delta$-cadinene $(3.11)[0.3 \%]$ & $\mathrm{N}$-methyl-N-nitro-methanamine (2.64) [0.4\%] & \\
\hline benzyl benzoate (5.95) [0.2\%] & methyl salicylate (3.11) $[0.3 \%]$ & \\
\hline oleic acid $(10.00)[0.2 \%]$ & $\alpha$-calacorene (3.48) $[0.3 \%]$ & \\
\hline $\begin{array}{l}\text { 4,4,8-trimethyltricyclo }[6.3 .1 .0(1,5)] \\
\text { dodecane-2,9-diol }(7.97)[0.2 \%]\end{array}$ & (Z)-3-hexen-1-ol, benzoate (4.06) [0.2\%] & \\
\hline benzoic acid (5.12) [0.2\%] & 4-benzyloxybenzoic acid (6.65) [0.2\%] & \\
\hline \multicolumn{3}{|c|}{ Trace compounds (i.e., $<0.1 \%$ ) } \\
\hline o-cymene (1.62) & oxalic acid, allyl isobutyl ester (1.68) & 2,3-pyridinedicarboxylic anhydride (1.78) \\
\hline 6-methyl-5-hepten-2-one (1.85) & eucalyptol (1.84) & 4-hexen-1-ol, acetate (1.98) \\
\hline $\begin{array}{l}\text { 2-oxo-4-phenyl-6-(4-chlorophenyl)-1,2- } \\
\text { dihydropyrimidine (1.87) }\end{array}$ & 1,3-dioxolan-2-one (1.92) & benzaldehyde (2.40) \\
\hline 1-methyl-3-(1-methylethenyl) benzene (2.08) & 1,1-diethoxy-ethane (2.03) & $\begin{array}{l}\text { 4a,8-dimethyl-2-(prop-1-en-2-yl)-1,2,3,4,4a,5,6,7- } \\
\text { octahydronaphthalene }(2.69)\end{array}$ \\
\hline propanoic acid, 2-methylpropyl ester (2.11) & 2-Pentyn-4-one (2.22) & dimethyl-silanediol (2.70) \\
\hline 4-hexen-1-ol, acetate (2.17) & 3-acetoxy-2-butanone (2.28) & azulene (2.72) \\
\hline$\alpha$-copaene (2.27) & 3-amino-butanoic acid (2.35) & 2,3,3-trimethyl-cyclobutanone (3.22) \\
\hline ammonium acetate (2.32) & propanoic acid, propyl ester (2.63) & $\begin{array}{l}\text { 2,6-bis(1,1-dimethylethyl)-2,5-Cyclohexadiene-1,4-dione } \\
\text { (3.23) }\end{array}$ \\
\hline linalool (2.44) & acetic anhydride (2.71) & acetic anhydride (3.63) \\
\hline benzoic acid, 2-hydroxy-, ethyl ester (3.25) & tetrahydro-2-(methoxymethyl)-furan (3.47) & 1H-pyrazolo[3,4-d]pyrimidin-4-amine $(3.70)$ \\
\hline phenylethyl alcohol (3.56) & butanoic acid, ethyl ester (4.04) & 3-methylpyridazine (3.79) \\
\hline a-calacorene (3.57) & 2-(formyloxy)-1-phenyl-ethanone (5.25) & neointermedeol (4.21) \\
\hline$\beta$-calacorene (3.70) & $\begin{array}{l}\text { 2-(4a,8-Dimethyl-1,2,3,4,4a,5,6,7-octahydro-naphthalen- } \\
\text { 2-yl)-prop-2-en-1-ol (5.32) }\end{array}$ & nonanoic acid (4.24) \\
\hline methyl octadecyl ether (5.69) & $\mathrm{N}$-phenyl-acetamide (5.62) & 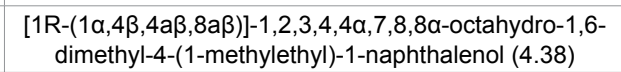 \\
\hline isospathulenol (5.73) & pentanedioic acid, (2,4-di-t-butylphenyl) mono-ester (5.74) & triethylene glycol (4.85) \\
\hline bicyclo[2.2.2]octane, 1,2,3,6-tetramethyl- (5.77) & phenanthrene (6.27) & 1-tetradecanol (4.88) \\
\hline 3-methyl-hexane (5.82) & 3-phenyl-1-propanol, acetate (7.14) & benzoic acid (5.10) \\
\hline 4-propylphenol (6.94) & (Z)-11-hexadecenoic acid (7.74) & 1-octadecanol (5.17) \\
\hline 1-nonadecene $(7.11)$ & $\begin{array}{c}\text { (4aS,7R)-7-(2-hydroxypropan-2-yl)-1,4a-dimethyl- } \\
4,4 \alpha, 5,6,7,8 \text {-hexahydronaphthalen-2(3H)-one }(7.78)\end{array}$ & benzophenone (5.32) \\
\hline oxalic acid, allyl octadecyl ester (8.89) & $\begin{array}{l}\text { 4,4,8-trimethyltricyclo[6.3.1.0(1,5)]dodecane-2,9-diol } \\
(7.91)\end{array}$ & 3,5-di-tert-butyl-4-hydroxybenzaldehyde (5.37) \\
\hline \multirow[t]{7}{*}{ octadecanoic acid (9.59) } & heptadecanoic acid (8.34) & vanillin (5.66) \\
\hline & 2-(3H)-furanone, dihydro-5-tetradecyl- $(8.90)$ & 2-ethyl-1-dodecanol (6.05) \\
\hline & & benzene, 1,1'-[1,2-ethanediylbis(oxy)]bis- (6.14) \\
\hline & & $\begin{array}{c}\text { 7,9-di-tert-butyl-1-oxaspiro(4,5)deca-6,9-diene-2,8-dione } \\
(6.48)\end{array}$ \\
\hline & & oleic acid, 3-hydroxypropyl ester (9.15) \\
\hline & & hexanamide (11.05) \\
\hline & & 1-methylene-2-vinylcyclopentane (11.94) \\
\hline 53 compounds & 53 compounds & 50 compounds \\
\hline
\end{tabular}

Table 1: Description and relative composition of fruit essential oils from two Psidium species and seasonal effect over the leaves' essential oil.

guava leaves during seasonal change as well. This further hint towards a general reduction in terpenes during rainy season (Table 1 and Figure 3B). Noteworthy, $\beta$-selinene [10] and (E)- $\beta$-caryophyllene [8] both share the same synthetic route as they appear to from the farnesyl cation forming thereafter the $(E, E)$-germacradenyl and $(E, E)$-humulyl cations, respectively [40]. We hypothesize that selinene isomers may be transformed into other important sesquiterpenes (e.g., Ref. [11] and Ref. [12]) during seasonal change. A similar phenomenon is observed as well in the case of $P$. friedrichsthalianum leaves (Figure 4A), in the latter, however linear aliphatic hydrocarbons are also increased 


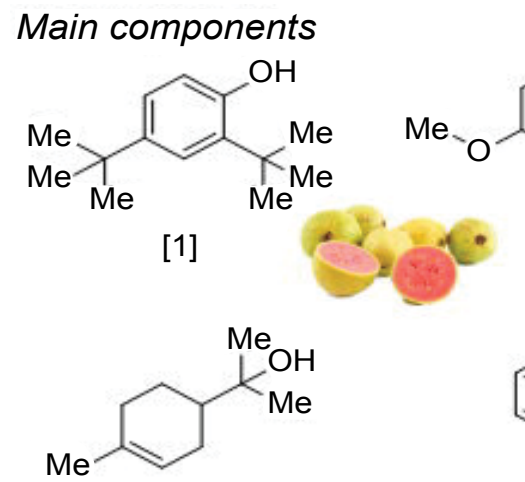

[2]<smiles>CC(C)C1CCC2(C)C(C)CCCC2(C)O1</smiles>

[3]<smiles>O=C(O)c1ccccc1</smiles>

[5]<smiles>C=CC(C)(O)CCC=C(C)CCC=C(C)C</smiles>

[7]<smiles>C=C1CC/C(C)=C\CC2CC(C)(C)[C@H]12</smiles>

[8]<smiles>O=C1C=CC(=C2CC3CCCCC23)C(=O)O1</smiles>

[6]

\section{Other compounds of interest}<smiles>C=C(C)[C@H]1CC[C@H](C)[C@]2(C)CCC=C(C)C12</smiles>

[9]<smiles>C=C(C)C1CCC(C)(C)C2CCCC(=C)C2C1</smiles>

[10]<smiles>C=C(CO)C1CCC2(C)CCCC(=C)C2C1</smiles>

[11]<smiles>CC1=CCCC2(C)CCC(C(C)(C)O)CC12</smiles>

[12]

Figure 1: Chemical structure of main components and noteworthy terpenoids from $P$. friedrichsthalianum and $P$. guajava fruits and leaves essential oil. Me used as abbreviated notation for the methylene moiety.

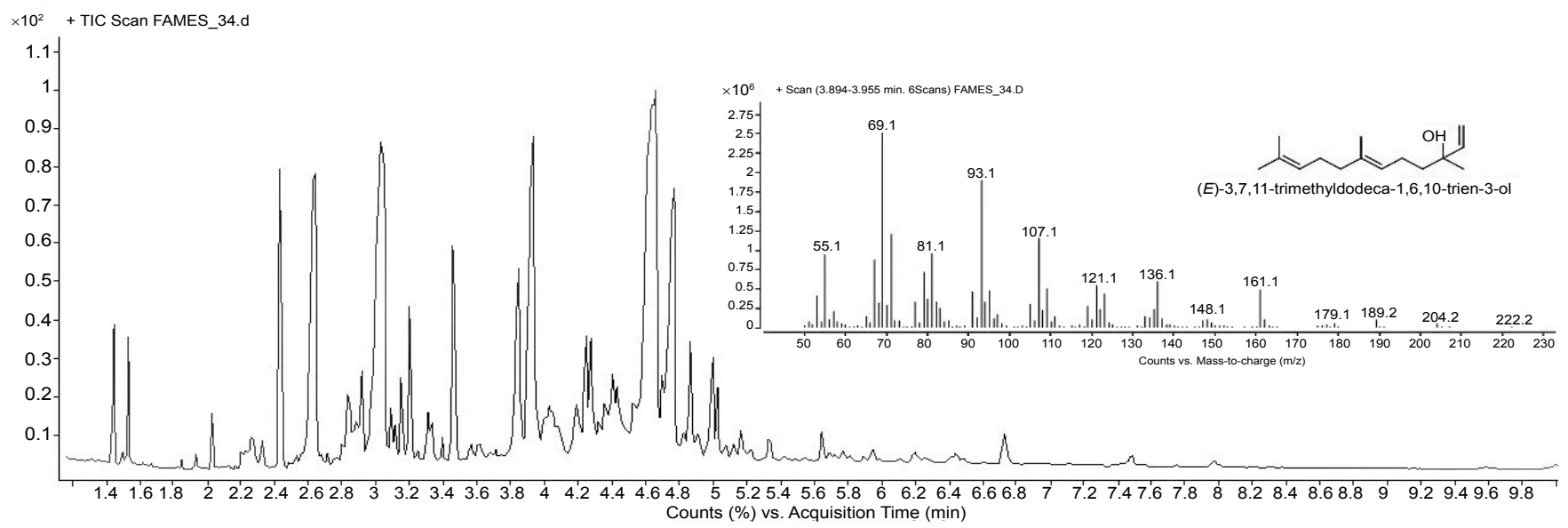

Figure 2: P. guajava fruit essential oil chromatogram and mass spectrum extraction for signal from 3.894 to 3.955 min corresponding to $\beta$-Nerolidol.

significantly ( $p<0.05$, Figure $4 \mathrm{~A})$. Worth mentioning is an increase in the concentration of $\beta$-selinene in the rainy season, that may be related to a growing insecticidal activity in the plant leaves [41,42] due, in turn, to a variation of insect population dynamics.

Principal component analysis demonstrated three clearly segregated subsets grouped by similarities in composition (Figure 5). Costa Rican guava (fruit) oil composition is the least similar from the rest of extracts (with main components including 2H-pyran-2,6-(3H)-dione, cis-13octadecanoic acid, $\alpha$-terpineol and $n$-hexadecanoic acid). In fact, these specific oils exhibited not only a stronger contrast, with respect of the rest of the study objects, but also display the highest diversity and number (i.e., 75 hits) of chemical identifiable compounds (Figure 5). 
Citation: Granados-Chinchilla F, Villegas E, Molina A, Arias C (2016) Composition, Chemical Fingerprinting and Antimicrobial Assessment of Costa Rican Cultivated Guavas (Psidium friedrichsthalianum (O. Berg) Nied. and Psidium guajava I.) Essential Oils from Leaves and Fruits. Nat Prod Chem Res 4: 236. doi:10.4172/2329-6836.1000236
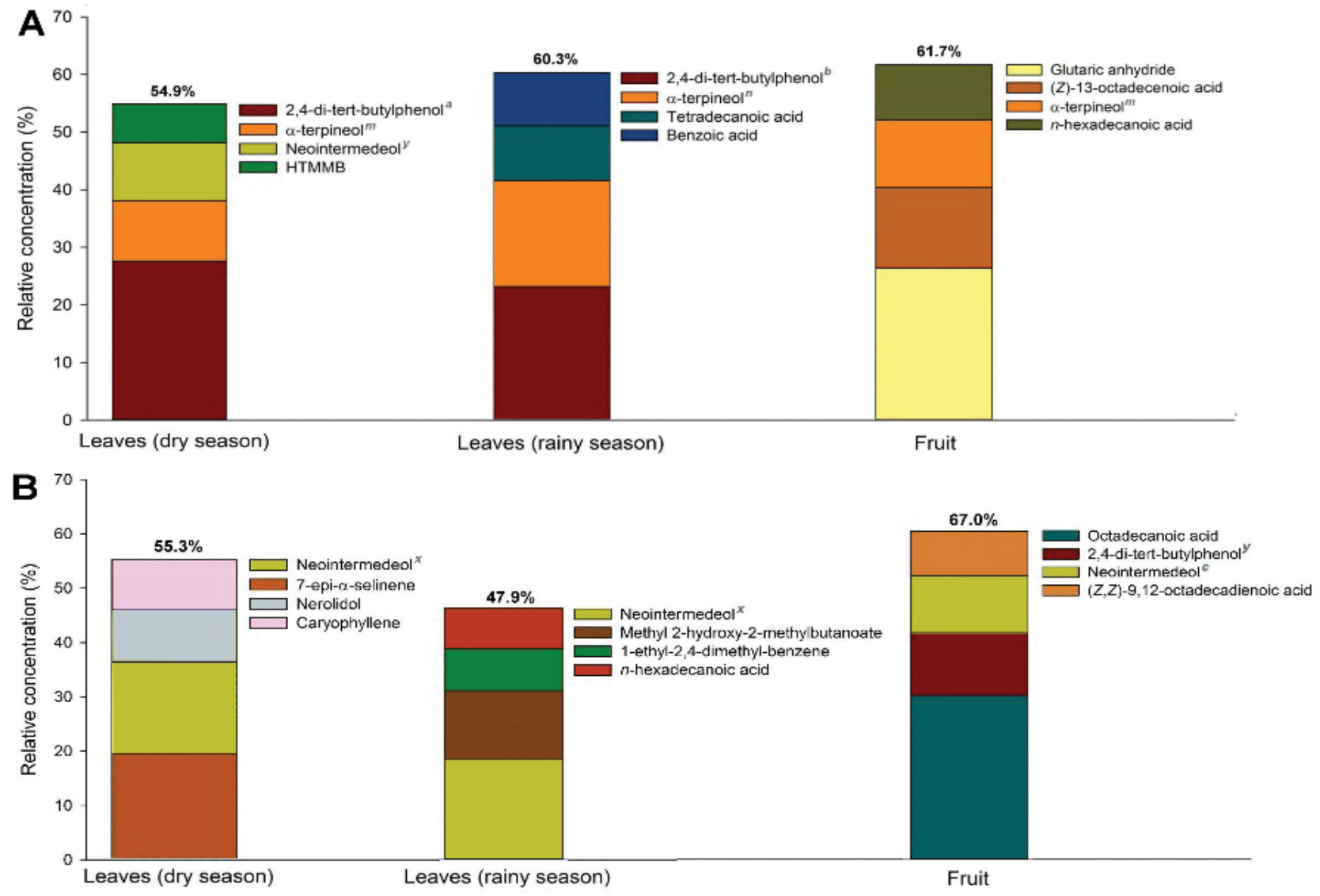

Figure 3: Comparison of essential oil main components among leaves and fruits from A. $P$. friedrichsthalianum and B. $P$. guajava. Dissimilar consecutive letters represent significant differences ( $p<0.05$, for all cases) among reiterated components. HTMMB: 2-hydroxy-3-(thiophen-2-yl)methyl-5-methoxy-1,4-benzoquinone.

\begin{tabular}{|c|c|c|c|c|c|c|c|}
\hline Strain (UFC mL-1)/Oila & \begin{tabular}{|c|}
$P$. \\
friedichsthalianum \\
leaves rainy \\
season
\end{tabular} & $\begin{array}{c}P . \\
\text { friedichsthalianum } \\
\text { leaves dry season }\end{array}$ & $\begin{array}{l}P . \text { guajava } \\
\text { leaves rainy } \\
\text { season }\end{array}$ & $\begin{array}{l}P . \text { guajava } \\
\text { leaves dry } \\
\text { season }\end{array}$ & $\begin{array}{c}P . \\
\text { friedichsthalianum } \\
\text { fruit }\end{array}$ & P. guajava fruit & $\begin{array}{c}\text { Oxytetracycline, } \\
10 \mu \mathrm{gL} \mathrm{m}^{-1}\end{array}$ \\
\hline \multicolumn{8}{|c|}{ Inhibition zone $\pm S D(\mathrm{~mm})$} \\
\hline S. Choleraesuis $\left(1.2 \times 10^{5}\right)$ & - & - & $44.7 \pm 0.5$ & - & $9.8 \pm 0.4$ & - & $21.7 \pm 1.7$ \\
\hline S. typhimurium $\left(4.0 \times 10^{4}\right)$ & - & - & $38.7 \pm 0.9$ & - & $9.8 \pm 0.7$ & - & $19.0 \pm 0.8$ \\
\hline S. enteritidis $\left(7.60 \times 10^{5}\right)$ & - & - & $42.7 \pm 1.9$ & - & $7.7 \pm 1.7$ & - & $20.7 \pm 0.5$ \\
\hline E. coli O157:H7 $\left(7.30 \times 10^{5}\right)$ & - & - & $44.5 \pm 1.9$ & - & $10.0 \pm 0.5$ & - & $29.0 \pm 0.8$ \\
\hline S. aureus $\left(7.00 \times 10^{4}\right)$ & - & - & $47.2 \pm 2.2$ & $8.3 \pm 0.4$ & $10.2 \pm 1.4$ & - & $33.7 \pm 1.2$ \\
\hline B. cereus $\left(2.70 \times 10^{5}\right)$ & $7.7 \pm 0.3$ & - & $37.3 \pm 4.1$ & $10.3 \pm 0.6$ & $10.0 \pm 1.4$ & - & $32.7 \pm 2.1$ \\
\hline B. subtillis $\left(1.49 \times 10^{6}\right)$ & - & $8.2 \pm 0.6$ & $34.0 \pm 2.2$ & $10.0 \pm 0.8$ & $9.7 \pm 1.7$ & $9.7 \pm 0.2$ & $14.3 \pm 0.5$ \\
\hline P. aeruginosa $\left(1.07 \times 10^{6}\right)$ & - & - & $36.8 \pm 1.4$ & - & $10.5 \pm 0.4$ & - & $9.0 \pm 1.6$ \\
\hline P. mirabilis $\left(7.60 \times 10^{5}\right)$ & - & - & $36.8 \pm 2.8$ & - & $10.7 \pm 0.8$ & - & $9.0 \pm 0.8$ \\
\hline Strain (UFC $\left.\mathrm{mL}^{-1}\right) / \mathrm{Oil}^{\mathrm{a}}$ & Limonene & Myrcene & Linalool & Eucalyptol & Thujone & \multicolumn{2}{|c|}{ Caryophyllene } \\
\hline \multicolumn{8}{|c|}{ Inhibition zone $\pm S D(\mathrm{~mm})$} \\
\hline S. choleraesuis $\left(1.2 \times 10^{5}\right)$ & - & - & $12.0 \pm 0.9$ & $9.7 \pm 0.3$ & $10.7 \pm 0.4$ & \multicolumn{2}{|c|}{-} \\
\hline S. typhimurium $\left(4.0 \times 10^{4}\right)$ & $4.2 \pm 2.2$ & - & $20.3 \pm 0.7$ & - & $9.0 \pm 0.8$ & \multicolumn{2}{|c|}{-} \\
\hline S. enteritidis $\left(7.60 \times 10^{5}\right)$ & - & - & $21.0 \pm 2.1$ & $10.0 \pm 0.5$ & $11.0 \pm 0.8$ & \multicolumn{2}{|c|}{-} \\
\hline E. coli O157:H7 $\left(7.30 \times 10^{5}\right)$ & - & - & $22.3 \pm 0.7$ & $8.2 \pm 0.2$ & $14.2 \pm 0.7$ & \multicolumn{2}{|c|}{-} \\
\hline S. aureus $\left(7.00 \times 10^{4}\right)$ & $5.3 \pm 1.5$ & $10.0 \pm 0.5$ & $15.0 \pm 1.2$ & - & $18.3 \pm 2.4$ & \multicolumn{2}{|c|}{$10.3 \pm 1.5$} \\
\hline B. cereus $\left(2.70 \times 10^{5}\right)$ & - & - & $38.3 \pm 1.2$ & - & $14.0 \pm 1.6$ & \multicolumn{2}{|c|}{$12.7 \pm 0.4$} \\
\hline B. subtillis $\left(1.49 \times 10^{6}\right)$ & $4.0 \pm 1.9$ & $8.7 \pm 0.4$ & $26.8 \pm 1.1$ & $9.8 \pm 0.3$ & $12.0 \pm 1.5$ & \multicolumn{2}{|c|}{$10.7 \pm 0.2$} \\
\hline P. aeruginosa $\left(1.07 \times 10^{6}\right)$ & - & - & - & - & - & \multicolumn{2}{|c|}{-} \\
\hline P. mirabilis $\left(7.60 \times 10^{5}\right)$ & - & - & $20.3 \pm 0.6$ & $9.3 \pm 0.6$ & $10.0 \pm 1.2$ & \multicolumn{2}{|c|}{$11.7 \pm 0.8$} \\
\hline
\end{tabular}

aDMSO (used as a negative control), terpinene and cymene oils did not exhibit any inhibition zones (i.e. 0 mm). ${ }^{\text {In }}$ hibition zones reported as the median of three replicates. Table 2: Antimicrobial activity for the recovered essential oils and some terpene standards. 
Citation: Granados-Chinchilla F, Villegas E, Molina A, Arias C (2016) Composition, Chemical Fingerprinting and Antimicrobial Assessment of Costa Rican Cultivated Guavas (Psidium friedrichsthalianum (O. Berg) Nied. and Psidium guajava I.) Essential Oils from Leaves and Fruits. Nat Prod Chem Res 4: 236. doi:10.4172/2329-6836.1000236

Page 9 of 10
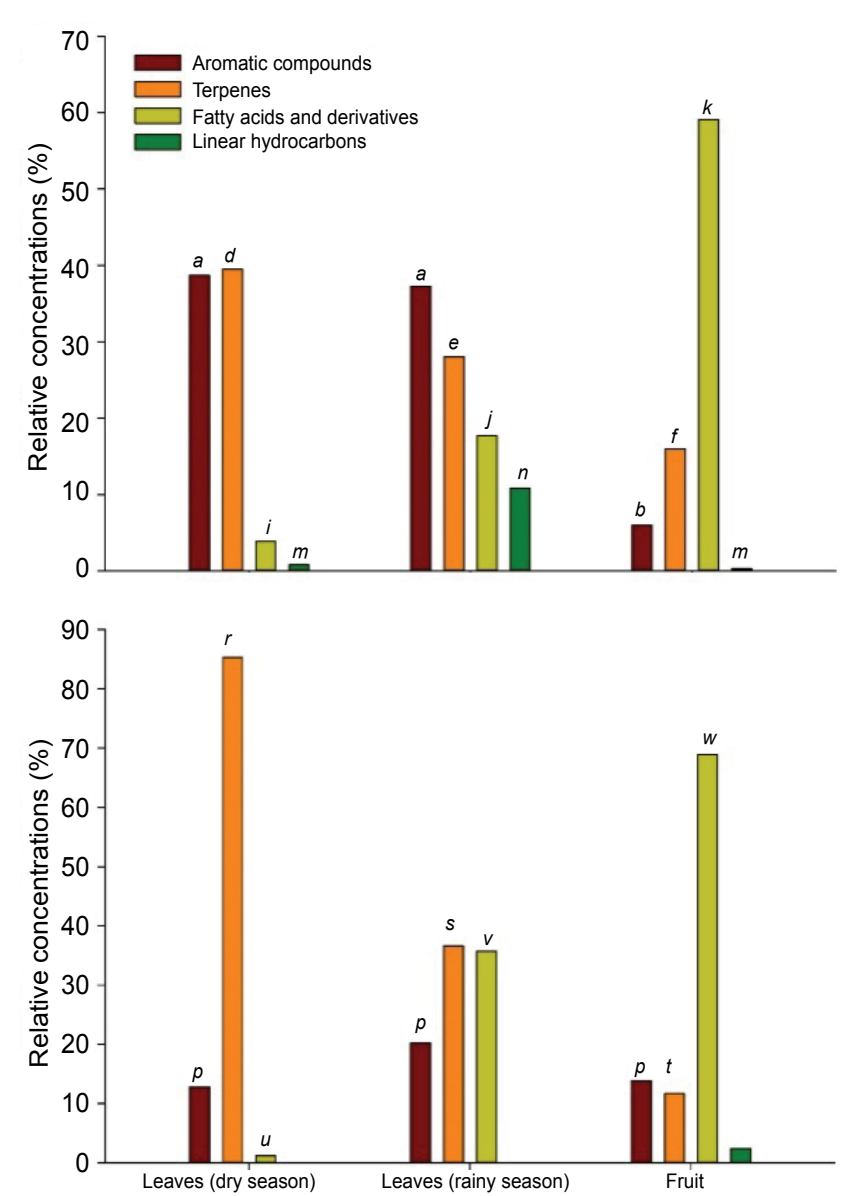

Figure 4: Comparison of essential oil grouped by structural families among leaves and fruits from A. P. friedrichsthalianum and B. P. guajava. Dissimila consecutive letters represent significant differences $(p<0.05$, for all cases) among reiterated components.

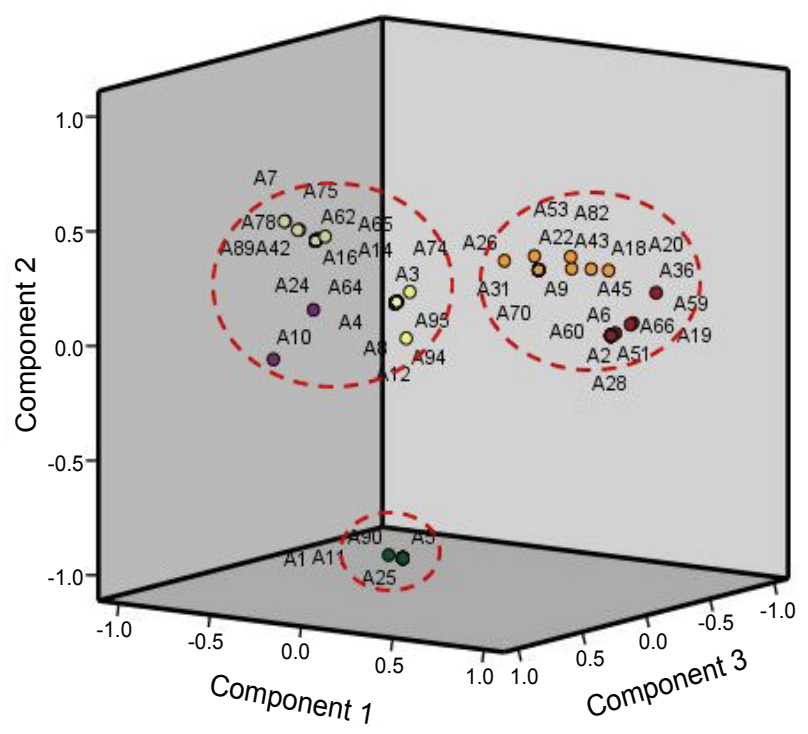

Figure 5: Component plot in rotated space for principal component analysis for the comparison of the six essential oils. $P$. guajava leaves rainy season , P. guajava leaves dry season $\ldots, P$. guajava fruit , $P$. friedrichsthalianum leaves rainy season $\square, P$. friedrichsthalianum leaves dry season,$P$ friedrichsthalianum fruit
As one may expect there are similarities among extracts from leaves obtained during dry and rainy season. Though, interestingly enough, important compositional differences are sufficient to distinguish them as well (Figure 5). The retention of some main compounds of importance such as 2,4-di-tert butylphenol [1] or $\alpha$-terpineol [2] may indicate some plant synthetic routes are conserved during normal climatic changes. Interestingly, in all cases leaf oils exhibited nearly the same amount of compounds (from 46 to 54 different hits, Table 1). Several of the terpenes and sesquiterpenes listed here-in have been reported in other tropical trees including other Myrtacea species [43].

Finally, the rainy season P. guajava leafs' oil is the most effective against the bacteria assayedexhibiting inhibition zones that ranged from 31 (B. subtilis) to $52 \mathrm{~mm}$ (S. aureus, Table 2) and was effective against both Gram-negative and Gram-positive bacteria which in turn, are food-spoilage related. This activity was significantly higher $(p<0.05)$ than that of the oxytetracycline $10 \mu \mathrm{g} \mathrm{mL}^{-1}$ solution (Table 2). Overall, $B$. cereus seems to be the more sensitive strain against all the essential oils producing inhibition zones from 0 to $42 \mathrm{~mm}$ (Table 2). A linalool oil standard exhibited a significantly stronger $(p<0.05)$ activity compared with the other essential oils tested producing inhibition zones from $0(P$. aeruginosa) to $40 \mathrm{~mm}$ (B. cereus, Table 2).

\section{Conclusion}

Both Costa Rican guava and guava major components may be segregated within families and, based on structural characteristics alone, seem to possess potential bioactive capacity. Climatic or seasonal changes seem to affect the overall composition of the leaf essential oil in both species, though some major components seem to prevail and only are modified concentration-wise. Similarities during chemical fingerprinting do arise when both species are compared. Furthermore, the tert-butyl moiety seems to be a conserved and extended throughout the volatile compounds in both species (present together in fruits and leaves). Finally, the effective antibacterial activity of the rainy season $P$. guajava leafs' oil, demonstrated here, should be further investigated to assess its potential as an alternative to conventional antibiotics. As part of future work, compounds responsible for eliciting bioactivity may be purified by analytical separation of the mixtures obtained.

\section{Acknowledgements}

Special thanks to Graciela Artavia for her help performing PCA analysis and loaning us some of the analytical standards used for chromatographic confirmation. We extend our appreciation to Guy Lamoureux, for his valuable comments during the drafting of the manuscript. We would like to thank Marisol Jiménez and Astrid Leiva for their excellent technical assistance. Vicerrectoría de Investigación supported this initiative by means of the project number B6257.

\section{References}

1. Mc Vaugh R (1968) The genera of American Myrtaceae: an interin report. Taxon 17: 354-418.

2. Dahlgren R, Thorne RF (1984) The order Myrtales: circumscription, variation and relationships. Ann. Missouri Bot Gard 71: 633-699.

3. Govaerts R, Sobral N, Ashton P, Barrie F, Holst BK, et al. (2008) World Checklist of Myrtaceae. Kew Publishing, Royal Botanic Gardens, Surrey, UK.

4. Rojas-Rodríguez F, Torres-Córdoba G (2013) Trees Central Valley of Costa Rica Cas reproduction (Psidium friedrichthalianum (Berg) Ndzu). Revista Forestal Mesoamericana Kourou 10: 30-31.

5. Segleau Earle J (2008) Árboles medicinales: el guayabo. Kurú: Revista Forestal 5: 1-3.

6. Djilas S, Čanadanović-Brunet J, Ćetković G (2009) By-products of fruits processing as a source of phytochemicals. Chemical Industry \& Chemical Engineering Quarterly 15: 191-202

7. Shaabana HAE, El-Ghoraba AH, Shibamoto T (2012) Bioactivity of essentia oils and their volatile aroma components: Review. J Essen Oil Res 24: 203-212. 
Citation: Granados-Chinchilla F, Villegas E, Molina A, Arias C (2016) Composition, Chemical Fingerprinting and Antimicrobial Assessment of Costa Rican Cultivated Guavas (Psidium friedrichsthalianum (O. Berg) Nied. and Psidium guajava I.) Essential Oils from Leaves and Fruits. Nat Prod Chem Res 4: 236. doi:10.4172/2329-6836.1000236

Page 10 of 10

8. IBP, Costa Rica Export-import Trade and Business Directory (2015) Edited by USA International Business Publications, Inc. Washington.

9. Govindasamy R, Arumugam S, Simon JE (2013) An Assessment of the Essential Oil and Aromatic Plant Industry with a Focus on Africa. African Natural Plant Products Volume II: Discoveries and Challenges in Chemistry, American Chemical Society, Washington.

10. Vekiari SA, Protopapadakis EE, Papadopoulou P, Papanicolaou D, Panou C, et al. (2002) Composition and seasonal variation of the essential oil from leaves and peel of a cretan lemon variety. J Agric Food Chem 50: 147-153.

11. Blanco Tirado C, StashenkoEE, Combariza MY, MartinezJR(1995)Comparative study of colombian citrus oils by high-resolution gas chromatography and gas chromatography-mass spectrometry. J Chrom A 697: 501-513.

12. Tucker AO, Maciarello MJ, Landrum LR (1995) Volatile leaf oils of American Myrtaceae III. Psidium cattleianum Sabine, P. friedrichsthalianum (Berg) Niedenzu, $P$. guajava L., Psidium guineense Sw., and Psidium sartorianum (Berg) Niedenzu. J Essent Oil Res 7: 187-190.

13. Chen HC, Sheu MJ, Lin LY, Wu CM (2007) Chemical composition of the leaf essential oil of Psidium guajava L. from Taiwan. J Essent Oil Res 19: 345-347.

14. Chalannavar RK, Venugopala KN, Baijnath H, Odhav B (2012) Chemical composition of essential oil of Psidium cattleianum var. lucidum (Myrtaceae). Afr J Biotechnol 11: 8341-8347.

15. Limberger RP, Farias FM, Sobral M, Zuanazzl JA, Henrtques AT (2001) Chemical composition of the volatile oil from Psidium cattleianum, Psidium guajava, Psidium incanum and Psidium luridum (Myrtaceae). Rev Bras Farm 82: 53-55.

16. Pichersky E, Gershenzon J (2002) The formation and function of plant volatiles: perfumes for pollinator attraction and defense. Curr Opin Plant Biol 5: 237-243.

17. Unsicker SB, Kunert G, Gershenzon J (2009) Protective perfumes: the role of vegetative volatiles in plant defense against herbivores. Curr Opin Plant Biol 12: $479-485$

18. Sharma A, Sehrawat SK, Singhrot RS, Tele A (2010) Morphological and Chemical Characterization of Psidium Species. Not Bot Hort Agrobot Cluj 38: 28-32.

19. Katiyar KP, Camacho Molina J, Matheus R (2000) Fruit flies (diptera: tephritidae) infesting fruits of the genus Psidium (Myrtaceae) and their altitudinal distribution in western Venezuela. Florida Entomologist 83: 480-486.

20. Li Y, Cracker LE, Potter T (1996) Effect of light level on essential oil of sage (Salvia officinalis)and thyme (Thymus vulgaris). Acta Horticulturae 426: 419-426.

21. Maffeia M, Scannerinia S (2000) UV-B Effect on Photomorphogenesys and Essential Oil Composition in Peppermint (Mentha piperita L.). J Essen Oil Res 12: $523-529$.

22. Feijó E, Oliveira R, Costa L (2014) Light affects Varronia curassavica essential oil yield by increasing trichomes frequency. Rev Bras Farmacogn 24: 516-523.

23. Dudai D, Putievsky E, Ravid U, Palevitch D, Halevy AH (1992) Monoterpene content in Origanum syriacum as affected by environmental conditions and flowering. Physiologia Plantarum 84: 453-459.

24. Zabaras D, Spooner-Hart R, Wyllie S (2002) Effects of mechanical wounding on concentration and composition of essential oil from Melaleuca alternifolia leaves. Biochemical Systematics and Ecology 30: 399-412.

25. Kulka M (1954) t-Butyl as a Blocking Group in the Synthesis of o-Hydroxybenzophenones. J Am Chem Soc 76: 5469-5471.

26. Batista R, de Jesús Silva Júnior A, de Oliveira AB (2009) Plant-Derived Antimalarial Agents: New Leads and Efficient Phytomedicines. Part II. NonAlkaloidal Natural Products. Molecules 14: 3037-3072.

27. Choi SJ, Kim JK, Kim HK, Harris K, Kim CJ, et al. (2013) 2,4-Di-tert-butylpheno from Sweet Potato Protects Against Oxidative Stress in PC12 Cells and in Mice. J Med Food 16: 977-983.
28. Amaral RR, Fernandes CP, Caramel OP, TietbohI LAC, Santos MG, et al. (2013) Essential Oils from Fruits with Different Colors and Leaves of Neomitranthes obscura (DC.) Silveira N: An Endemic Species from Brazilian Atlantic Forest. BioMed Research International 2013: 1-7.

29. Cuvelier ME, Richarda H, Berseta C (1992) Comparison of the Antioxidative Activity of Some Acid-phenols: Structure-Activity Relationship. Biosci Biotech Biochem 56: 324-325.

30. Amala VE, Jeyaraj M (2014) Determination of antibacterial, antifungal, bioactive constituents of Triphala by FT-IR and GC-MS analysis. International Journal of Pharmacy and Pharmaceutical Sciences 6: 123-126.

31. Hassan SB, Gali-Muhtasib H, Göransson H, Larsson R (2010) Alpha Terpineol: A Potential Anticancer Agent Which Acts through Suppressing NF-kB Signalling. Anticancer Research 30: 1911-1920.

32. Souza R, Cardoso M, Menezes C, Silva J, De Sousa D, et al. (2011) Gastroprotective activity of a-terpineol in two experimental models of gastric ulcer in rat. Daru 19: 277-281.

33. Sell C (2012) Chemistry of Essential oils Handbook of Essential Oils. In Handbook of Essential Oils Science, Technology and Applications. Baser KHC Buchbauer G (eds.), CRC Press, New York, USA, pp: 126-127.

34. Padovan A, Keszei A, Külheim C, Foley WJ (2013) The evolution of foliar terpene diversity in Myrtaceae. Phytochemistry Reviews 13: 695-716.

35. FDA (United States Food and Drug Administration) (2015) Last accessed June $30^{\text {th }} 2016$.

36. Adrian JA, Arancon NQ, Mathews BW, Carpenter JR (2012) Proximate analysis, in vitro organic matter digestibility, and energy content of common guava ( $P$ sidium guajava L.) and yellow, strawberry guava ( $P$ sidium cattleianum Var. lucidum) tree parts and fruits as potential forage. J Agric Food Chem 60: 10398-10405.

37. Mahmoud R, Ibrahim D, Badawi M (2003) Effect of supplementation of broiler diets with guava leaves and/or olive oil on growth, blood metabolites and immune response. Benha Veterinary Medical Journal 25: 23-32.

38. Shen SC, Cheng FC, Wu NJ (2008) Effect of Guava (Psidium guajava Linn.) Leaf Soluble Solids on Glucose Metabolism in Type 2 Diabetic Rats. Phytother Res 22: 1458-1464.

39. Aharoni A, Giri AP, Verstappen FWA, Bertea CM, Sevenier R, et al. (2004) Gain and Loss of Fruit Flavor Compounds Produced by Wild and Cultivated Strawberry Species. Plant Cell 16: 3110-3131.

40. Degenhardt J, Köllner TG, Gershenzon J (2009) Monoterpene and sesquiterpene synthases and the origin of terpene skeletal diversity in plants. Phytochemistry 70: 1621-1637.

41. Zoubiri S, Baaliouamer A (2014) Potentiality of plants as source of insecticide principles. Journal of Saudi Chemical Society 18: 925-938.

42. Chu S, Jiang G, Liu Z (2011) Insecticidal compounds from the essential oil of Chinese medicinal herb Atractylodes chinensis. Pest Manag Sci 67: 1253-1257.

43. Courtois EA, Baraloto C, Paine CE, Petronelli P, Blandinieres PA, et al. (2012) Differences in volatile terpene composition between the bark and leaves of tropical tree species. Phytochemistry $82: 81-88$. 